\title{
Dead-time-modified photocount mean and variance for chaotic radiation
}

\author{
Giovanni Vannucci* and Malvin Carl Teich \\ Columbia Radiation Laboratory, Department of Electrical Engineering, Columbia University, New York, \\ New York 10027
}

Received August 19, 1980

\begin{abstract}
General expressions are obtained for the mean and variance of the number of events in a fixed but arbitrary sampling time for a nonparalyzable dead-time counter. The input is assumed to be a Poisson point process whose rate is a stochastic process, and the dead time is assumed to be small in comparison with the fluctuation time of the driving process. The mean is shown to depend only on the first-order statistics of the rate, whereas the variance is formally shown to depend on both the first- and the second-order statistics of the rate. For the particular process arising in the detection of chaotic light, an explicit expression is obtained for the dependence of the dead-timemodified variance on the power spectrum of the radiation. It is demonstrated that the variance takes on a particularly simple form for chaotic light with Lorentzian and Gaussian spectra. In the regime in which our study is valid, it turns out that the dead-time dependence of the variance is contained in a multiplicative function that is essentially independent of the spectral properties of the light.
\end{abstract}

\section{INTRODUCTION}

The probability distribution for a dead-time-modified pulse counter ${ }^{1,2}$ has been studied by a number of researchers in a broad variety of disciplines, such as photon counting, ${ }^{3-9} \mathrm{nu}$ clear counting, ${ }^{8-12}$ and neural counting.,8,13-17 Many cases have been studied in detail, including paralyzable and nonparalyzable counting under blocked, unblocked, and equilibrium conditions. Attention has also been given to the gradual recovery (sick-time) system ${ }^{15}$ and to the variable dead-time case. ${ }^{2,14}$ Müller has summarized the results of a number of authors ${ }^{11,12}$ and has compiled a comprehensive, although by now somewhat dated, bibliography on dead-time effects. ${ }^{18}$

Although most of the work cited above is applicable only when the input to the counter is a Poisson point process with constant rate, a few results are also available for the case in which the rate is not constant. Cantor and Teich, ${ }^{4}$ Teich and McGill, ${ }^{5}$ and Bédard ${ }^{3}$ present expressions for the photoncounting distribution when the intensity of the light is a random process, with the sampling time much smaller than the coherence time of the light.

In a previous publication, ${ }^{19}$ we obtained expressions for the mean and the variance of the number of events in a fixed sampling time for a nonparalyzable dead-time counter when the input process is Poisson with a rate that is a known function of time. In this paper we extend those results to the case in which the rate of the input process is an arbitrary stochastic process, under the constraint that it vary slowly with respect to the duration of the dead time. No constraints on the sampling time are imposed. We show how the dead-timemodified mean and variance depend on the first- and second-order statistics of the rate. In the context of the detection of light, we obtain explicit expressions for the photocount mean and variance for chaotic radiation of arbitrary spectral properties. Although the magnitude of the variance depends explicitly on the power spectrum of the radiation in the absence of dead time, it is shown that the decrease in variance with increasing dead time is basically spectrum independent. Analytical and graphical results for the dead-time-modified mean and variance are provided for chaotic light of Lorentzian and Gaussian spectra.

\section{DEAD-TIME-MODIFIED MEAN}

Consider a Poisson counting process whose instantaneous rate is a known function of time that we denote by $\lambda(t)[\lambda(t) \geq 0] .{ }^{20}$ Let this process be the input to a nonparalyzable dead-time counter, i.e., a counter that does not record pulses during a time interval of fixed duration $\tau_{d}$ after recording a given pulse (the pulses that arrive at the input to the counter during this dead time are lost). We consider the case for which the counter is always connected to the input process; this is the equilibrium counter as opposed to the blocked or unblocked counter. Actually, in the limits where our results are applicable, the number of pulses recorded during a sampling time is $\gg 1$, and therefore the differences among blocked, unblocked, and equilibrium counters become negligible, so that our results are indeed valid for all three types of counter. We are concerned with the statistics of the number of pulses $n$ counted in a certain time interval $\left(t_{v}, t_{0}+T\right)$. The expected number of pulses during this interval is given by Eq. (3) of Ref. 19 ,

$$
E[n]=\int_{t_{0}}^{t_{0}+T} \frac{\lambda(t)}{1+\lambda(t) \tau_{d}} \mathrm{~d} t,
$$

under the constraint that $\lambda(t)$ vary slowly enough to be virtually constant for any time interval with duration of the order of $\tau_{d}$.

If the instantaneous rate of the Poisson counting process is not a known function of time but is itself a random process, 
we observe that each realization of the random process will be a well-defined function of time. Therefore, for a given realization $\lambda$ of the random process, we can regard Eq. (1) as a conditional expectation and write

where $\lambda(t) \equiv \lambda$.

$$
E[n \mid \lambda]=\int_{t_{0}}^{t_{0}+T} \frac{\lambda(t)}{1+\lambda(t) \tau_{d}} \mathrm{~d} t
$$

The conditional expectation is itself a random variable, and its expected value corresponds to the unconditional expectation. ${ }^{21}$ Thus

$$
\begin{array}{r}
E[n]=E[E[n \mid \lambda]]=E\left[\int_{t_{0}}^{t_{0}+T} \frac{\lambda(t)}{1+\lambda(t) \tau_{d}} \mathrm{~d} t\right] \\
=\int_{t_{0}}^{t_{0}+T} E\left[\frac{\lambda(t)}{1+\lambda(t) \tau_{d}}\right] \mathrm{d} t,
\end{array}
$$

where the expectation is taken with respect to the statistics of $\lambda$. If the random process to which $\lambda$ belongs is stationary, then the integrand in Eq. (3) is independent of $t$, and we can write

$$
E[n]=T E\left[\frac{\lambda(t)}{1+\lambda(t) \tau_{d}}\right]=T \int_{0}^{\infty} \frac{\lambda}{1+\lambda \tau_{d}} p(\lambda) \mathrm{d} \lambda ;
$$

here $\lambda(t)$ is a random variable, since it represents the value of the random process at a given instant $t$ (first-order statistics), and $p(\lambda)$ is its probability density function.

The constraint that $\lambda(t)$ vary slowly with respect to $\tau_{d}$, as indicated earlier (see Ref. 19, Section 2), will be satisfied by almost all realizations of the random process if and only if the coherence time $\tau_{\mathrm{c}}$ of the rate satisfies the condition

$$
\tau_{c} \gg \tau_{d},
$$

since the coherence time provides a measure of the time scale over which rate variations occur. ${ }^{6}$ Therefore, for Eq. (4) to be applicable, the condition expressed by Eq. (5) must be satisfied.

\section{DEAD-TIME-MODIFIED VARIANCE}

Equation (13) of Ref. 19 gives an expression for the deadtime-modified variance when the rate is a known function of time:

$$
\operatorname{var}[n]=\int_{t_{0}}^{t_{0}+T} \frac{\lambda(t)}{\left[1+\lambda(t) \tau_{d}\right]^{3}} \mathrm{~d} t
$$

We observe that

$$
E\left[n^{2}\right]=\operatorname{var}[n]+\{E[n]\}^{2},
$$

and therefore when the rate is a random process of which $\lambda \equiv$ $\lambda(t)$ is a realization, we can follow the same argument presented in the previous section to arrive at

$$
\begin{aligned}
E\left[n^{2} \mid \lambda\right]= & \operatorname{var}[n \mid \lambda]+\{E[n \mid \lambda]\}^{2} \\
= & \int_{t_{0}}^{t_{0}+T} \frac{\lambda(t)}{\left[1+\lambda(t) \tau_{d}\right]^{3}} \mathrm{~d} t \\
& \quad+\left\{\int_{t_{0}}^{t_{0}+T} \frac{\lambda(t)}{1+\lambda(t) \tau_{d}} \mathrm{~d} t\right\}^{2} .
\end{aligned}
$$

By evaluating the expectation of Eq. (8), we obtain

$$
\begin{aligned}
E\left[n^{2}\right]=E\left[\int_{t_{0}}^{t_{0}+T} \frac{\lambda(t)}{\left[1+\lambda(t) \tau_{d}\right]^{3}} \mathrm{~d} t\right] & \\
& +E\left[\left\{\int_{t_{0}}^{t_{0}+T} \frac{\lambda(t)}{1+\lambda(t) \tau_{d}} \mathrm{~d} t\right\}^{2}\right] .
\end{aligned}
$$

The first term on the right-hand side of Eq. (9) can be manipulated in the same way as Eq. (3), whereas in the second term the integral raised to the second power can be rewritten as a double integral. Thus

$$
\begin{aligned}
& E\left[n^{2}\right]=T \int_{0}^{\infty} \frac{\lambda}{\left(1+\lambda \tau_{d}\right)^{3}} p(\lambda) \mathrm{d} \lambda \\
& +E\left[\int_{t_{0}}^{t_{0}+T} \cdot \int_{t_{0}}^{t_{0}+T} \frac{\lambda(t)}{1+\lambda(t) \tau_{d}} \cdot \frac{\lambda\left(t^{\prime}\right)}{1+\lambda\left(t^{\prime}\right) \tau_{d}} \mathrm{~d} t \mathrm{~d} t^{\prime}\right],
\end{aligned}
$$

valid if the random process is stationary. By bringing the expectation operator inside the double integral, the second term in Eq. (10) can be expressed as

$$
\int_{t_{0}}^{t_{0}+T} \int_{t_{0}}^{t_{0}+T} \eta\left(\left|t-t^{\prime}\right|\right) \mathrm{d} t \mathrm{~d} t^{\prime}
$$

where

$$
\eta\left(\left|t-t^{\prime}\right|\right)=E\left[\frac{\lambda(t)}{1+\lambda(t) \tau_{d}} \cdot \frac{\lambda\left(t^{\prime}\right)}{1+\lambda\left(t^{\prime}\right) \tau_{d}}\right] .
$$

This notation is permissible only for a stationary random process, in which case the expected value in Eq. (12) depends only on the difference between $t$ and $t^{\prime}$. With an appropriate change of integration variables (such as $\tau=t-t^{\prime}$ and $\tau^{\prime}=t$ $+t^{\prime}$ ), the double integral in Eq. (11) can be simplified to a single integral:

$$
\int_{t_{0}}^{t_{0}+T} \int_{t_{0}}^{t_{0}+T} \eta\left(\left|t-t^{\prime}\right|\right) \mathrm{d} t \mathrm{~d} t^{\prime}=2 \int_{0}^{T}(T-\tau) \eta(\tau) \mathrm{d} \tau .
$$

We can now write an expression for the dead-time-modified variance when the rate is a random process, by using the results of Eqs. (4), (7), (10), and (13):

$$
\begin{aligned}
\operatorname{var}[n]= & E\left[n^{2}\right]-\{E[n]\}^{2} \\
= & T \int_{0}^{\infty} \frac{\lambda}{\left(1+\lambda \tau_{d}\right)^{3}} p(\lambda) \mathrm{d} \lambda \\
& +2 \int_{0}^{T}(T-\tau) \eta(\tau) \mathrm{d} \tau \\
& -\left[T \int_{0}^{\infty} \frac{\lambda}{1+\lambda \tau_{d}} p(\lambda) \mathrm{d} \lambda\right]^{2} .
\end{aligned}
$$

The evaluation of $\eta(\tau)$ requires knowledge of the secondorder statistics of the rate, i.e., the joint probability density for two values of the rate separated by a time interval $\tau$. In fact, the explicit expression for $\eta(\tau)$ is

$$
\eta(\tau)=\int_{0}^{\infty} \int_{0}^{\infty} \frac{\lambda_{1}}{1+\lambda_{1} \tau_{d}} \cdot \frac{\lambda_{2}}{1+\lambda_{2} \tau_{d}} p\left(\lambda_{1}, \lambda_{2} ; \tau\right) \mathrm{d} \lambda_{1} \mathrm{~d} \lambda_{2}
$$

where $p\left(\lambda_{1}, \lambda_{2} ; \tau\right)$ is the joint probability density function.

The constraints under which Eq. (6) was obtained (as discussed in Ref. 19) are somewhat more restrictive than those for Eq. (1). Hence, for Eq. (14) to be valid, in addition to the condition expressed in Eq. (5), the following condition must also be satisfied: 


$$
\tau_{c} \gg\left(\bar{\lambda} \tau_{d}\right)^{2} \tau_{d} / 6
$$

where $\bar{\lambda}$ is the average value of the rate. This is equivalent to the condition expressed in Eq. (11) of Ref. 19 for the case in which the rate is a known function of time.

\section{DEAD-TIME-MODIFIED MEAN FOR CHAOTIC RADIATION}

The evaluation of Eq. (4) for the photon-counting detection of chaotic radiation of arbitrary spectrum is straightforward, since the first-order statistics do not depend on the spectrum. It is well-known that for linearly polarized chaotic radiation incident upon a detector substantially smaller than the coherence area, the probability density function for the rate $\lambda$ is given by ${ }^{6}$

$$
p(\lambda)=(1 \bar{\lambda}) e^{-\lambda / \lambda}
$$

where $\bar{\lambda}$ is the average value of $\lambda$. By using Eq. (17), Eq. (4) becomes

$$
E[n]=T \int_{0}^{\infty} \frac{\lambda}{1+\lambda \tau_{d}} \frac{1}{\bar{\lambda}} e^{-\lambda / \bar{\lambda}} \mathrm{d} \lambda .
$$

The substitutions $x=\lambda \bar{\lambda}$ and $y=x+1 / \bar{\lambda} \tau_{d}$ yield

$$
\begin{aligned}
E[n] & =\frac{T}{\tau_{d}}\left(\int_{0}^{\infty} e^{-x} \mathrm{~d} x-\frac{e^{1 / \bar{\lambda} \tau_{d}}}{\bar{\lambda} \tau_{d}} \int_{1 / \bar{\lambda} \tau_{d}}^{\infty} \frac{e^{-y}}{y} \mathrm{~d} y\right) \\
& =\frac{\bar{\lambda} T}{\bar{\lambda} \tau_{d}}\left[1-\frac{e^{1 / \bar{\lambda} \tau_{d}}}{\bar{\lambda} \tau_{d}} E_{1}\left(1 / \bar{\lambda} \tau_{d}\right)\right],
\end{aligned}
$$

where

$$
\begin{array}{r}
E_{m}(z)=\int_{1}^{\infty} \frac{e^{-z t}}{t^{m}} \mathrm{~d} t=z^{m-1} \int_{z}^{\infty} \frac{e^{-t}}{t^{m}} \mathrm{~d} t, \\
m=0,1,2, \ldots,
\end{array}
$$

is the (tabulated) exponential-integral function. ${ }^{22}$

In Fig. 1 we present a plot of the theoretical counting efficiency, which is the normalized mean $E[n] / \bar{\lambda} T$, as a function of $\bar{\lambda} \tau_{d}$. The solid curve represents the result for a Poisson

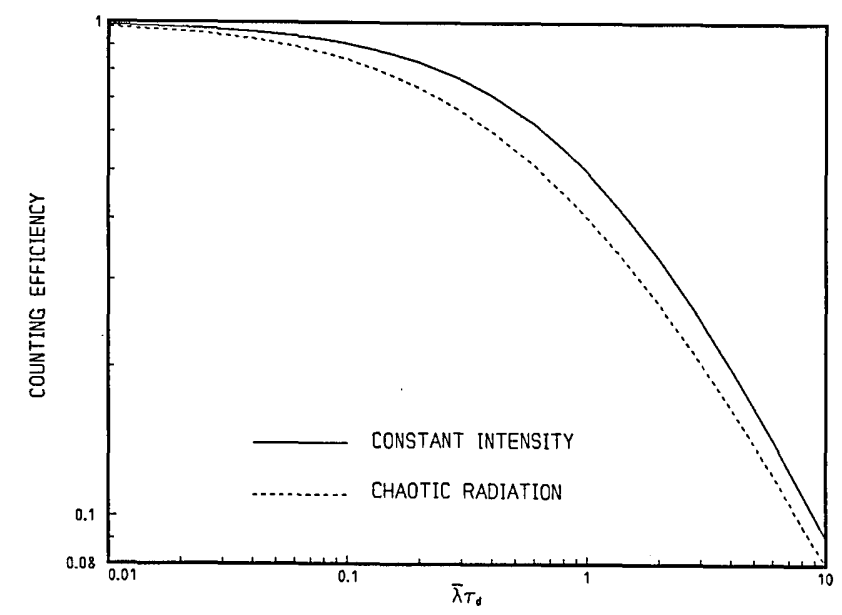

Fig. 1. Counting efficiency $(E[n] / \bar{\lambda} T)$ versus $\bar{\lambda} \tau_{d}$, where $\bar{\lambda}$ is the average driving rate and $\tau_{d}$ is the dead time. Curves are for a Poisson process in which the rate is constant (solid curve) and for chaotic radiation of arbitrary spectrum (dashed curve). It is clear that the efficiency is significantly reduced (up to $24 \%$ ) when the rate is not constant. The results are analogous to those presented in Fig. 1 of Ref. 19 for a rate that is a known function of time. point process with constant rate $\left(1 / 1+\bar{\lambda} \tau_{d}\right)$ (see Fig. 1 of Ref. 19). The dashed curve is the result for chaotic radiation of arbitrary spectrum [Eq. (19)]; it lies everywhere below the solid curve in accordance with the upper limit on dead-time counter efficiency derived in Section 6 of Ref. 19.

\section{DEAD-TIME-MODIFIED VARIANCE FOR CHAOTIC RADIATION}

The evaluation of the variance, which is expressed in Eq. (14), is more complex. The third term on the right-hand side of Eq. (14), which is the square of the mean, is obtained directly from Eq. (19):

$$
\begin{aligned}
& {\left[T \int_{0}^{\infty} \frac{\lambda}{1+\lambda \tau_{d}} p(\lambda) \mathrm{d} \lambda\right]^{2}} \\
& =\left\{\frac{\bar{\lambda} T}{\bar{\lambda} \tau_{d}}\left[1-\frac{e^{1 / \bar{\lambda} \tau_{d}}}{\bar{\lambda} \tau_{d}} E_{1}\left(1 / \bar{\lambda} \tau_{d}\right)\right]\right\}^{2} .
\end{aligned}
$$

The first term on the right-hand side of Eq. (14) is dealt with rather easily. Inserting Eq. (17) provides

$T \int_{0}^{\infty} \frac{\lambda}{\left(1+\lambda \tau_{d}\right)^{3}} p(\lambda) \mathrm{d} \lambda=T \int_{0}^{\infty} \frac{\lambda}{\left(1+\lambda \tau_{d}\right)^{3}} \frac{1}{\bar{\lambda}} e^{-\lambda / \bar{\lambda}} \mathrm{d} \lambda$.

The form of Eq. (21) is closely related to the form of Eq. (18), and the same technique is used to evaluate the integral. Again we let $x=\lambda \bar{\lambda}$ and $y=x+1 \bar{\lambda} \tau_{d}$. Then we use the exponential-integral function defined in Eq. (20), together with the recurrence relation ${ }^{22} E_{m+1}(z)=m^{-1}\left[e^{-z}-z E_{m}(z)\right]$, valid for $m=1,2,3, \ldots$, to obtain

$$
\begin{aligned}
& T \int_{0}^{\infty} \frac{\lambda}{\left(1+\lambda \tau_{d}\right)^{3}} \frac{1}{\bar{\lambda}} e^{-\lambda / \bar{\lambda}} \mathrm{d} \lambda \\
& \quad=\bar{\lambda} T\left(\frac{1}{\bar{\lambda} \tau_{d}}\right)^{2} e^{1 / \bar{\lambda} \tau_{d}}\left[E_{2}\left(\frac{1}{\bar{\lambda} \tau_{d}}\right)-E_{3}\left(\frac{1}{\bar{\lambda} \tau_{d}}\right)\right] \\
& =\frac{\bar{\lambda} T}{2\left(\bar{\lambda} \tau_{d}\right)^{3}}\left[\left(1+\bar{\lambda} \tau_{d}\right)-\left(1+2 \bar{\lambda} \tau_{d}\right) \frac{e^{1 / \bar{\lambda} \tau_{d}}}{\bar{\lambda} \tau_{d}} E_{1}\left(\frac{1}{\bar{\lambda} \tau_{d}}\right)\right] .
\end{aligned}
$$

Evaluation of the middle term of Eq. (14) requires a specific knowledge of the second-order statistics of the radiation that, in the case of chaotic light, is uniquely determined by the power spectrum. This is carried out as follows.

It is well known ${ }^{23}$ that for narrow-band chaotic radiation, the radiation field can be decomposed into the sum of in-phase and quadrature components at the center frequency $\omega_{0}$, where the two components are Gaussian random processes with identical statistics. Their autocorrelation and cross-correlation functions $R(\tau)$ are, respectively,

$$
\begin{aligned}
R_{y y}(\tau)=R_{x x}(\tau)=E[x(t+\tau) x(t)] & \\
& =\int_{-\infty}^{\infty} s^{2}\left(\omega_{0}+\omega\right) \cos \omega \tau \mathrm{d} \omega
\end{aligned}
$$

and

$$
R_{x y}(\tau)=E[x(t+\tau) y(t)]=\int_{-\infty}^{\infty} s^{2}\left(\omega_{0}+\omega\right) \sin \omega \tau \mathrm{d} \omega
$$

where $s^{2}(\omega)$ corresponds to the power spectrum of the radiation, $\omega_{0}$ is the center radian frequency, and $x(t)$ and $y(t)$ are the in-phase and quadrature components of the radiation field, respectively. 
For narrow-band chaotic radiation, the power spectrum can be represented by a function $s^{2}(\omega)$ that is significantly different from zero only in a region around $\omega_{0}$ that is small with respect to $\omega_{0}$. Under these conditions we see from the expressions for $R_{x x}(\tau)$ and $R_{x y}(\tau)$ given in Eq. (23) that $x(t)$ and $y(t)$ will be slowly varying with respect to $\omega_{0}$, and the intensity of the radiation will therefore be given by

$$
I(t)=C^{\prime}\left[x^{2}(t)+y^{2}(t)\right],
$$

where $C^{\prime}$ is a constant. Accordingly, when a detector is exposed to this type of radiation, photon absorptions at the input of the detector (before dead time) will occur as the events of a Poisson point process whose rate is the random process

$$
\lambda(t)=C\left[x^{2}(t)+y^{2}(t)\right],
$$

where $C$ is again a constant.

To evaluate Eq. (15), we require an expression for the joint probability density function of the values of $\lambda$ at two points in time separated by a specified interval $\tau$. This result was obtained by Barrett and Lampard in 1955 [see Eq. (76) of Ref. 24]. In our notation it is written as

$$
\begin{aligned}
& p\left(\lambda_{1}, \lambda_{2} ; \tau\right)=\frac{1}{\overline{\lambda^{2}\left[1-R^{2}(\tau)\right]}} I_{0}\left\{\frac{2 R(\tau) \sqrt{\lambda_{1} \lambda_{2}}}{\overline{\bar{\lambda}}\left[1-R^{2}(\tau)\right]}\right\} \\
& \times \exp \left\{-\frac{\lambda_{1}+\lambda_{2}}{\bar{\lambda}\left[1-R^{2}(\tau)\right]}\right\},
\end{aligned}
$$

where $I_{0}\{x\}$ is the modified Bessel function of order zero and

$$
\begin{aligned}
\bar{\lambda} & =E\left[\lambda_{1}\right]=E\left[\lambda_{2}\right]=E\left[C\left(x_{1}^{2}+y_{1}^{2}\right)\right]=2 C R_{x x}(0), \\
R^{2}(\tau) & =\frac{R_{x x}^{2}(\tau)+R_{y y}^{2}(\tau)}{R_{x x}^{2}(0)}=\frac{\left|\int_{-\infty}^{\infty} e^{-i \omega \tau} s^{2}\left(\omega_{0}+\omega\right) \mathrm{d} \omega\right|^{2}}{\left[\int_{-\infty}^{\infty} s^{2}(\omega) \mathrm{d} \omega\right]^{2}} \\
& =\frac{\int_{-\infty}^{\infty} \int_{-\infty}^{\infty} s^{2}\left(\omega_{1}\right) s^{2}\left(\omega_{2}\right) \cos \left(\omega_{1}-\omega_{2}\right) \mathrm{d} \omega_{1} \mathrm{~d} \omega_{2}}{\left[\int_{-\infty}^{\infty} s^{2}(\omega) \mathrm{d} \omega\right]^{2}}
\end{aligned}
$$

We see that $R(\tau)$ can be expressed in terms of the power spectrum of the radiation; since $s^{2}(\omega) \geq 0$ for any value of $\omega$, Eq. (28) shows that $R^{2}(\tau) \leq 1$ for any value of $\tau$, and thus the expression $1-R^{2}(\tau)$, which appears in Eq. (26), is always nonnegative. $\mathrm{R}^{2}(\tau)$ is in the form of a normalized intensity correlation function minus 1 .

As is indicated in Eq. (78) of Ref. 24, we can now make use of the following identity,

$$
\begin{aligned}
(1-t)^{-1} \exp \left[-(x+y) \frac{t}{1-t}\right] & I_{0}\left\{\frac{2 \sqrt{x y t}}{1-t}\right\} \\
= & 1+\sum_{n=1}^{\infty} t^{n} L_{n}(x) L_{n}(y),
\end{aligned}
$$

where $L_{n}(x)$ is the Laguerre polynomial of order $n$, to rewrite Eq. (26) in the form

$$
\begin{aligned}
& p\left(\lambda_{1}, \lambda_{2} ; \tau\right) \\
& \quad=p\left(\lambda_{1}\right) p\left(\lambda_{2}\right)\left[1+\sum_{n=1}^{\infty} R^{2 n}(\tau) L_{n}\left(\lambda_{1} / \bar{\lambda}\right) L_{n}\left(\lambda_{2} / \bar{\lambda}\right)\right] .
\end{aligned}
$$

By substituting Eq. (30) into Eq. (15) and exchanging the summation with the double integral, we obtain

$$
\begin{aligned}
\eta(\tau) & =\left[\int_{0}^{\infty} \frac{\lambda}{1+\lambda \tau_{d}} p(\lambda) \mathrm{d} \lambda\right]^{2} \\
& +\sum_{n=1}^{\infty} R^{2 n}(\tau)\left[\int_{0}^{\infty} \frac{\lambda / \bar{\lambda}}{1+\lambda \tau_{d}} e^{-\lambda / \lambda} L_{n}(\lambda / \bar{\lambda}) \mathrm{d} \lambda\right]^{2} .
\end{aligned}
$$

The first term on the right-hand side of Eq. (31) is simply the square of the mean divided by $T$, and the expression in brackets in the summation does not depend on $\tau$, so Eq. (31) can be rewritten as

$$
\eta(\tau)=\{E[n] / T\}^{2}+\bar{\lambda}^{2} \sum_{n=1}^{\infty} a_{n}^{2}\left(\bar{\lambda} \tau_{d}\right) R^{2 n}(\tau),
$$

where

$$
a_{n}\left(\bar{\lambda} \tau_{d}\right)=\int_{0}^{\infty} \frac{x L_{n}(x)}{1+\bar{\lambda} \tau_{d} x} e^{-x} \mathrm{~d} x
$$

Since $^{22}$

$$
L_{n}(x)=\sum_{k=0}^{n}(-1)^{k}\left(\begin{array}{l}
n \\
k
\end{array}\right) \frac{1}{k !} x^{k},
$$

we can evaluate the ratio of the two polynomials in the integrand of Eq. (33) to obtain

$$
a_{n}\left(\bar{\lambda} \tau_{d}\right)=\int_{0}^{\infty}\left(\frac{b_{n}}{1+\bar{\lambda} \tau_{d} x}+\sum_{j=0}^{n} b_{n, j} x^{j}\right) e^{-x} \mathrm{~d} x,
$$

where

$$
\begin{gathered}
b_{n, j}=(-1)^{j} \sum_{k=j}^{n}\left(\begin{array}{l}
n \\
k
\end{array}\right) \frac{1}{k !}\left(\bar{\lambda} \tau_{d}\right)^{j-k-1}, \\
b_{n}=-b_{n, 0}=-\left(1 / \bar{\lambda} \tau_{d}\right) L_{n}\left(-1 / \bar{\lambda} \tau_{d}\right) .
\end{gathered}
$$

Evaluating the integral in Eq. (34) yields

$$
\begin{aligned}
a_{n}\left(\bar{\lambda} \tau_{d}\right) & =b_{n} \int_{0}^{\infty} \frac{e^{-x}}{1+\bar{\lambda} \tau_{d} x} \mathrm{~d} x+\sum_{j=0}^{n} b_{n, j} \int_{0}^{\infty} x^{j} e^{-x} \mathrm{~d} x \\
& =b_{n}\left(1 / \bar{\lambda} \tau_{d}\right) e^{1 / \bar{\lambda} \tau_{d}} E_{1}\left(1 / \bar{\lambda} \tau_{d}\right)+\sum_{j=0}^{n} b_{n, j}(j !) .
\end{aligned}
$$

Substituting the values of $b_{n}$ and $b_{n, j}$ given by Eq. (35) into Eq. (36) gives

$$
\begin{aligned}
a_{n}\left(\bar{\lambda} \tau_{d}\right) & =\sum_{k=0}^{n}\left(\begin{array}{l}
n \\
k
\end{array}\right) \frac{\left(\bar{\lambda} \tau_{d}\right)^{-k-2}}{k !} \\
& \times\left[\sum_{j=0}^{k}(j !)(-1)^{\left.j\left(\bar{\lambda} \tau_{d}\right)^{j+1}-e^{1 / \bar{\lambda} \tau_{d}} E_{1}\left(1 / \bar{\lambda} \tau_{d}\right)\right]}\right.
\end{aligned}
$$

Finally, substituting Eqs. (22), (32), and (37) into Eq. (14) allows us to write a complete expression for the dead-timemodified variance for chaotic radiation,

$$
\begin{aligned}
\operatorname{var}[n]= & \frac{\bar{\lambda} T}{2\left(\bar{\lambda} \tau_{d}\right)^{3}}\left[\left(1+\bar{\lambda} \tau_{d}\right)-\left(1+2 \bar{\lambda} \tau_{d}\right) \frac{e^{1 / \bar{\lambda} \tau_{d}}}{\bar{\lambda} \tau_{d}} E_{1}\left(\frac{1}{\bar{\lambda} \tau_{d}}\right)\right] \\
& +2 \bar{\lambda}^{2} \sum_{n=1}^{\infty} a_{n}^{2}\left(\bar{\lambda} \tau_{d}\right) \int_{0}^{T}(T-\tau) R^{2 n}(\tau) \mathrm{d} \tau
\end{aligned}
$$

Equation (38a) shows explicitly that the expression for the dead-time-modified variance given in Eq. (14) depends on the power spectrum of the radiation.

For $\bar{\lambda} \tau_{d}=0$, Eq. (33) can be directly evaluated to yield $a_{1}(0)$ $=1$ and $a_{n}(0)=0$, for $n>1$. In that case, Eq. (38a) reduces to 


$$
\operatorname{var}[n]=\bar{\lambda} T+2 \bar{\lambda}^{2} \int_{0}^{T}(T-\tau) R^{2}(\tau) \mathrm{d} \tau
$$

which is the known expression for the chaotic-light photocount variance in the absence of dead time. ${ }^{6}$ As is well understood, light sources with different power spectra give rise to different variances under the same conditions. What is interesting and new here is that the functional dependency of the variance on the dead time turns out to be virtually independent of the power spectrum, as will be demonstrated in the next section.

\section{VARIANCE FOR CHAOTIC RADIATION WITH LORENTZIAN AND GAUSSIAN SPECTRA}

We evaluate Eq. (38a) for the Lorentzian and Gaussian cases. The Lorentzian spectrum $G(\omega)$ is given by

$$
G(\omega)=\frac{A}{\Gamma^{2}+\left(\omega-\omega_{0}\right)^{2}},
$$

so that

$$
R(\tau)=e^{-\mathrm{\Gamma}|\tau|},
$$

where $1 / \Gamma$ is the coherence time ${ }^{6}$ and $A$ is a constant. With this substitution, the second term in Eq. (38a) becomes

$$
\begin{aligned}
2 \bar{\lambda}^{2} & \sum_{n=1}^{\infty} a_{n}^{2}\left(\bar{\lambda} \tau_{d}\right) \int_{0}^{T}(T-\tau) \exp (-2 n \Gamma \tau) \mathrm{d} \tau \\
\quad= & \frac{(\bar{\lambda} T)^{2}}{(\Gamma T)^{2}} \sum_{n=1}^{\infty}\left[a_{n}^{2}\left(\bar{\lambda} \tau_{d}\right) / 2 n^{2}\right][2 n \Gamma T+\exp (-2 n \Gamma T)-1]
\end{aligned}
$$

For a Gaussian spectrum,

$$
G(\omega)=A^{\prime} \exp \left[-\left(\omega-\omega_{0}\right)^{2} / 2 \Gamma^{2}\right]
$$

and

$$
R(\tau) \doteq \exp \left(-\Gamma^{2} \tau^{2} / 2\right) .
$$

Again, $1 / \Gamma$ is the coherence time. By using this substitution, the second term in Eq. (38a) becomes

$$
\begin{aligned}
2 \bar{\lambda}^{2} & \sum_{n=1}^{\infty} a_{n}^{2}\left(\bar{\lambda} \tau_{d}\right) \int_{0}^{T}(T-\tau) \exp \left(-n \Gamma^{2} \tau^{2}\right) \mathrm{d} \tau \\
= & \frac{(\bar{\lambda} T)^{2}}{(\Gamma T)^{2}} \sum_{n=1}^{\infty}\left[a_{n}^{2}\left(\bar{\lambda} \tau_{d}\right) / n\right]\{\sqrt{\pi n} \Gamma T[1-\operatorname{erfc}(\sqrt{n} \Gamma T)] \\
& \left.-1+e^{-n(\Gamma T)^{2}}\right\} .
\end{aligned}
$$

Here $\operatorname{erfc}(x)$ denotes the complementary error function ${ }^{22}$

$$
\operatorname{erfc}(x)=\frac{2}{\sqrt{\pi}} \int_{x}^{\infty} e^{-t^{2}} \mathrm{~d} t .
$$

We have numerically calculated the photocount variance [Eq. (38a)] for light with these spectral properties for a variety of values of the parameters $\Gamma T, \bar{\lambda} T$, and $\bar{\lambda} \tau_{d}$. The results were obtained with the help of an IBM 370 computer and are summarized in Figs. 2 and 3.

The solid curve in Fig. 2 displays the ratio of the variance to the unmodified mean $[\operatorname{var}[n] / \bar{\lambda} T]$ as a function of $\bar{\lambda} \tau_{d}$ for a Poisson process with constant rate. In this case,$^{19}$

$$
\frac{\operatorname{var}[n]}{\bar{\lambda} T}=\frac{1}{\left(1+\bar{\lambda} \tau_{d}\right)^{3}},
$$

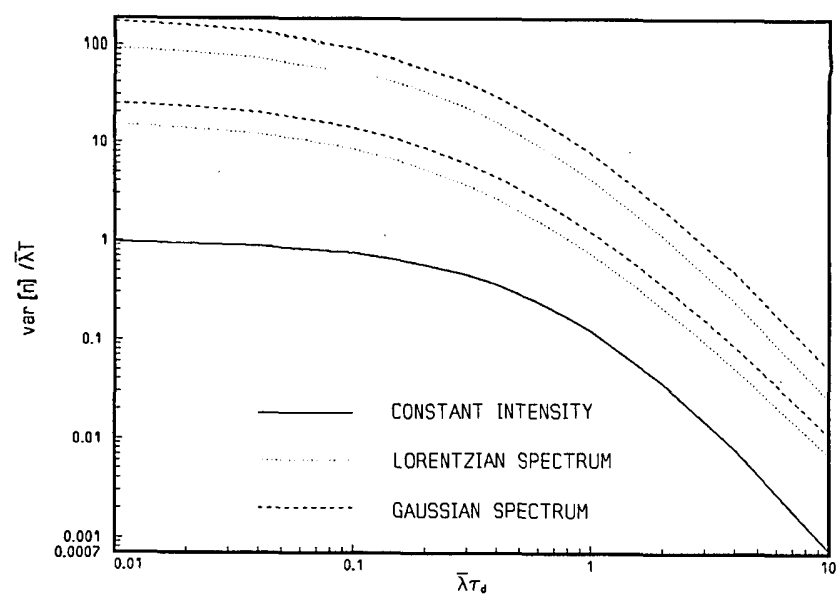

Fig. 2. Ratio of dead-time-modified variance to unmodified mean $(\operatorname{var}[n] / \bar{\lambda} T)$ versus $\bar{\lambda} \tau_{d}$ for a Poisson process with constant rate (solid curve), for chaotic radiation with Gaussian spectrum (dashed curves), and for chaotic radiation with Lorentzian spectrum (dotted curves). The values of the parameters for the chaotic radiation curves are $\Gamma T$ $=100, \bar{\lambda} T=10,000$ (upper curves) and $\Gamma T=2, \bar{\lambda} T=40$ (lower curves). The vertical separation between any of the curves for chaotic radiation is essentially independent of $\bar{\lambda} \tau_{d}$, an indication that the different curves are scaled versions of one another. Thus the normalized variance will be essentially the same for all four curves, as is demonstrated in Fig. 3.

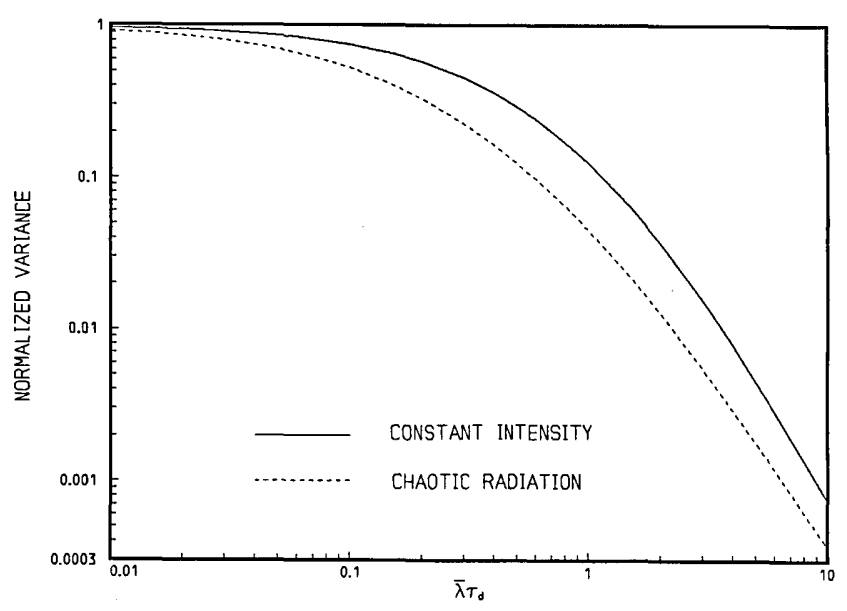

Fig. 3. Normalized variance (ratio of dead-time-modified variance to unmodified variance) versus $\bar{\lambda} \tau_{d}$. Curves are for a Poisson process with constant rate (solid curve) and for chaotic radiation (dashed curve). All results were obtained under the assumption that the dead time is much shorter than the coherence time $\left(\tau_{d} \ll 1 / \Gamma\right)$, in which case the effect of dead time turns out to be virtually independent of spectral shape (see text). The normalized variance is therefore the same function of $\bar{\lambda} \tau_{d}$ for all types of chaotic radiation. The results are similar in character to those presented in Fig. 2 of Ref. 19 for a rate that is a known function of time.

which is independent of the value of the mean. The other curves represent the results for chaotic light with Gaussian spectrum (dashed curves) and Lorentzian spectrum (dotted curves), with $\Gamma T=100, \bar{\lambda} T=10,000$ (upper curves) and $\Gamma T$ $=2, \bar{\lambda} T=40$ (lower curves). It is clear from Fig. 2 that the vertical separation between any of the dashed and dotted curves (chaotic radiation) is reasonably independent of $\bar{\lambda} \tau_{d}$, particularly where $\bar{\lambda} \tau_{d}$ is not too large. Since the ordinate is a logarithmic scale, this implies that the ratio is constant and therefore that the normalized variance, i.e., the ratio of the 
dead-time-modified variance to the variance in the absence of dead time, will be the same for the two spectra, for the two sets of parameter values. Thus it appears that under the conditions for which our results are applicable, the effect of dead time on the variance is independent of the spectral shape. Stated differently, the normalized variance is a prescribed function of $\bar{\lambda} \tau_{d}$, essentially independent of spectral parameters. We have obtained curves for several sets of parameters, for both Gaussian and Lorentzian spectra, and we have found this to be consistently true.

The behavior, which is not a priori obvious, can be understood by observing that our results are applicable only when the dead time is much shorter than the coherence time $\left(\tau_{d} \ll\right.$ $1 / \Gamma)$. For sufficiently small $\tau_{d}$, the rate of the Poisson process can be locally approximated as being constant. Thus the dead-time-modified point process has a rate that is locally a function of the unmodified rate only and as such is not influenced by the second-order statistical properties of the radiation.

Figure 3 provides a plot of the normalized dead-timemodified variance, i.e., the ratio of the variance in the presence of dead time to the variance in the absence of dead time, for a Poisson point process with constant rate (solid curve) and for chaotic radiation (dashed curve). The latter is reasonably independent of the spectral characteristics of the light, as was discussed above.

A few words about the procedure used to evaluate Eq. (38a) by computer are in order. The infinite summation must be truncated at a point where the neglected terms cause a sufficiently small error. For values of $\bar{\lambda} \tau_{d}$ larger than 1 , several $a_{n}\left(\bar{\lambda} \tau_{d}\right)$ terms are needed to achieve acceptable accuracy. The expression for $a_{n}\left(\bar{\lambda} \tau_{d}\right)$ [Eq. (37)] includes summations of terms with alternating signs that almost precisely cancel and must therefore be evaluated with great precision to achieve acceptable accuracy. To obtain the data for the figures, we used extended precision (REAL $* 16)$ in our calculations. The exponential integral function $E_{1}(x)$ was evaluated to REAL $* 16$ precision through its series expansion ${ }^{22}$ for $x<$ 2 and through its continued fraction expansion ${ }^{22}$ for $x \geq 2$; other functions (exponential, erfc, etc.) were provided by the FORTRAN $\mathrm{H}$ compiler with full REAL $* 16$ accuracy.

\section{CONCLUSIONS}

The principal results of this paper comprise analytic expressions, complemented by computer results, for the mean and variance of a dead-time-modified Poisson counting process driven by a continuous rate process representative of chaotic radiation. It has been assumed that the nonparalyzable dead time is small in comparison with the coherence time (inverse bandwidth) of the light. In this limit, as well as in the absence of dead time, the photocount mean is explicitly shown to depend only on the first-order statistics of the radiation. As was expected, the counting efficiency lies below that for a constant intensity source for all values of the dead-time parameter $\bar{\lambda} \tau_{d}$.

The photocount variance, on the other hand, exhibits a strong dependence on the spectral properties of the radiation even in the absence of dead time. The forms of the deadtime-modified analytic expressions for the cases of Lorentzian and Gaussian spectra are therefore substantially different. Nevertheless, numerical computer evaluation of these ex- pressions (variance as a function of $\bar{\lambda} \tau_{d}$ ) demonstrates that the effect of dead time on the variance is essentially independent of the spectral shape of the light, although a dependence can be discerned to develop at higher values of $\bar{\lambda} \tau_{d}$. This can be understood by noting that our results are applicable only when the dead time is much shorter than the coherence time $\left(\tau_{d} \ll 1 / \Gamma\right)$. For sufficiently small $\tau_{d}$, the rate of the Poisson process can be locally approximated as being constant so that the dead-time-modified point process has a rate that is locally a function of the unmodified rate only and as such is not influenced by the second-order statistical properties of the radiation.

In light of our results, we may expect that the dead-timemodified count variance will exhibit an increasing dependence on the spectrum as $\tau_{d}$ becomes larger; indeed, this dependence should become substantial as $\tau_{d}$ approaches $1 / \Gamma$. An analysis in that regime, although difficult to carry out, might therefore lead to the intriguing possibility of using a dead-time counter to extract spectroscopic information about a radiation source. The notion of dead-time spectroscopy is not unlike photocounting spectroscopy, ${ }^{6}$ but the upper limit of bandwidths that could be measured by the technique would be $\sim 10 \mathrm{GHz}$. This value is higher than the upper limit for photocounting spectroscopy, $\sim 100 \mathrm{MHz}$, because the dead time can in principle be made about a factor of 100 smaller than the 10-nsec minimum counting time in which variations can be detected with commercially available fast electronic circuits. The region of usefulness of dead-time spectroscopy would probably be similar to that of the Fabry-Perot étalon.

Finally, we point out that the dead-time-modified count mean may also depend on the spectral properties of the light for $\tau_{d} \sim 1 / \Gamma$, in which case dead-time spectroscopy could be implemented quite easily. Kikkawa et al. ${ }^{25,26}$ have calculated the efficiency (normalized mean) of a dead-time-modified photon counter for Lorentzian light and have discussed the dependence of this quantity on spectral parameters. Their results are valid only in the limit where the coherence time is much smaller than the mean time interval between pulses, however, and this is not a useful region [see remarks following Eq. (10) in Ref. 25].

This work was supported by the Joint Services Electronics Program (U.S. Army, U.S. Navy, and U.S. Air Force) under contract DAAG29-79-C-0079 and by the National Science Foundation under grant ENG78-26498. We are grateful to John Brown of Pennsylvania State University for bringing the work of Barrett and Lampard to our attention.

* Present address, Bell Laboratories, Holmdel, New Jersey 07733.

\section{REFERENCES}

1. W. Feller, "On probability problems in the theory of counters," in Studies and Essays: A Volume for the Anniversary of Courant (Wiley, New York, 1948), p. 105.

2. E. Parzen, Stochastic Processes (Holden-Day, San Francisco, 1962), pp. 117-186.

3. G. Bédard, "Dead-time corrections to the statistical distribution of photoelectrons," Proc. Phys. Soc. 90, 131-141 (1967).

4. B. I. Cantor and M. C. Teich, "Dead-time-corrected photocounting distributions for laser radiation," J. Opt. Soc. Am. 65, 786-791 (1975).

5. M. C. Teich and W. J. McGill, "Neural counting and photon counting in the presence of dead time," Phys. Rev. Lett. 36, 754-758, 1473 (1976). 
6. B. Saleh, Photoelectron Statistics (Springer-Verlag, Berlin/ Heidelberg/New York, 1978), pp. 101-102, 116, 175, 272-279.

7. M. C. Teich and G. Vannucci, "Olservation of dead-time-mudified photocounting distributions for modulated laser radiation," J. Opt. Soc. Am. 68, 1338-1342 (1978).

8. M. C. Teich and B. I. Cantor, "Information, error, and imaging in deadtime-perturbed doubly-stochastic Poisson counting systems," IEEE J. Quantum Electron. QE-14, 993-1003 (1978).

9. G. Vannucci and M. C. Teich, "Equivalence of threshold detection with and without dead time," Appl. Opt. 18, 3886-3887 (1979).

10. I. DeLotto, P. F. Manfredi, and P. Principi, "Counting statistics and dead-time losses, Part 1," Energ. Nucl. (Milan) 11, 557-564 (1964).

11. J. W. Müller, "Dead-time problems," Nucl. Instrum. Methods 112, 47-57 (1973).

12. J. W. Müller, "Some formulae for a dead-time-distorted Poisson process," Nucl. Instrum. Methods 117, 401-404 (1974).

13. L. M. Ricciardi and F. Esposito, "On some distribution functions for non-linear switching elements with finite dead time," Kybernetik 3, 148-152 (1966).

14. M. C. Teich, L. Matin, and B. I. Cantor, "Refractoriness in the maintained discharge of the cat's retinal ganglion cell," J. Opt. Soc. Am. 68, 386-402 (1978).

15. M. C. Teich and P. Diament, "Relative refractoriness in visual information processing," Biol. Cybern., to be published.

16. M. C. Teich and G. Lachs, "A neural counting model incorporating refractoriness and spread of excitation. I. Application to intensity discrimination," J. Acoust. Soc. Am. 66, 1738-1749 (1979).

17. G. Lachs and M. C. Teich, "A neural counting model incorporating refractoriness and spread of excitation. II. Application to loudness estimation," J. Acoust. Soc. Am. 68, to be published (1980).

18. J. W. Maller, ed., "Bibliography on dead time efrects," Bureau International des Poids et Mesures, Sèvres, France, Report No. BIPM-75/6, 1975 (unpublished).

19. G. Vannucci and M. C. Teich, "Effects of rate variation on the counting statistics of dead-time-modified Poisson processes," Opt. Commun. 25, 267-272 (1978).

20. D. L. Snyder, Random Point Processes (Wiley-Interscience, New York, 1975), p. 38.

21. A. Papoulis, Probability, Random Variables and Stochastic Processes (McGraw-Hill, New York, 1965), pp. 178, 208.

22. M. Abramowitz and I. A. Stegun, eds., Handbook of Mathematical Functions with Formulas, Graphs, and Mathematical Tables, Nat. Bur. Stand. (U.S.) Appl. Math. Ser., U.S. Government Printing Office, Washington, D.C., 1964 (Dover, New York, 1965): Chap. 3.10.1, p. 19; Eqs. 5.1.1, 5.1.4, p. 228; Eqs. 5.1.11, 5.1.14, 5.1.22, p. 229; Eq. 7.1 .2 , p. 297 ; Eq. 22.3 .9 , p. 775

23. G. Vannucci and M. C. Teich, "Computer simulation of superposed coherent and chaotic radiation," Appl. Opt. 19, 548-553 (1980).

24. J. F. Barrett and D. G. Lampard, "An expansion for some second-order probability distributions and its application to noise problems," IRE Trans. Inf. Theory IT-1, 10-15 (1955).

25. A. Kikkawa, K. Ohkubo, H. Sato, and N. Suzuki, "Photoelectric pulse interval distribution of Gaussian-Lorentzian light," Opt. Commun. 12, 227-230 (1974).

26. A. Kikkawa, K. Ohkubo, T. Yoshimura, and N. Suzuki, "Statistical properties of Gaussian-Lorentzian light and dead time loss of photon-counting system," Jpn. J. Appl. Phys. 13, 1226-1231 (1974). 